\title{
Globe
}

Revue internationale d'études québécoises

\section{Forum social québécois. Poutine altermondialiste à la sauce québécoise?}

\section{Thomas Chiasson-Lebel}

Volume 12, numéro 1, 2009

Coopération et missonnariat

URI : https://id.erudit.org/iderudit/1000773ar

DOI : https://doi.org/10.7202/1000773ar

Aller au sommaire du numéro

Éditeur(s)

Globe, Revue internationale d'études québécoises

ISSN

1481-5869 (imprimé)

1923-8231 (numérique)

Découvrir la revue

Citer cet article

Chiasson-Lebel, T. (2009). Forum social québécois. Poutine altermondialiste à la sauce québécoise? Globe, 12(1), 133-147. https://doi.org/10.7202/1000773ar d'utilisation que vous pouvez consulter en ligne.

https://apropos.erudit.org/fr/usagers/politique-dutilisation/ 


\section{PERSPECTIVE}

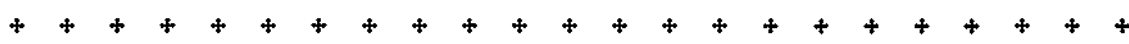 \\ FORUM SOCIAL \\ QUÉBÉCOIS. POUTINE \\ ALTERMONDIALISTE \\ À LA SAUCE QUÉBÉCOISE ?}

\section{THOMAS CHIASSON-LEBEL' \\ Université du Québec à Montréal}

$+++++4++++++++++++++++++$

La première édition du Forum social québécois (FSQ) s'est terminée le 26 août 2007, et les membres du comité organisateur se félicitaient par voie de communiqué de la réussite de cet événement. En lisant ce document, on comprend que le succès est mesuré à la quantité de participants, d'activités réalisées et de propositions formulées. On y trouvait cette phrase qui résume bien le propos: "Il s'agissait du plus gros rassemblement de la gauche de l'histoire du $Q u e ́ b^{2}{ }^{2}$ ». S'il y a sans doute là une marque de réussite, est-elle suffisante? Dans cet article, nous tenterons d'évaluer, à l'aune des principes fondateurs du Forum social mondial, si le FSQ s'est bel et bien inscrit dans la démarche des forums sociaux comme il

$$
+4
$$

1. L'auteur a participé à l'organisation de l'édition vénézuélienne du Forum social mondial polycentrique de 2006 et aux activités du Forum social québécois de septembre 2007.

2. Cité dans Alexandre SHIELD, "Le premier Forum social québécois a mobilisé 5000 participants", Le Devoir, 27 août 2007, p. A2. 
le devait, et si des améliorations ne pourraient pas être apportées aux prochaines éditions.

\section{LA RAISON D'ETRE DES FORUMS SOCIAUX}

Pour contrer l'avancée mondiale du néolibéralisme, des organisations et mouvements sociaux à l'échelle de la planète ont lancé depuis quelques années une nouvelle forme d'initiative. Prenant le nom de "forum social", celle-ci s'inscrit en continuité et en rupture avec le grand courant d'internationalisation de la lutte contre le capitalisme qui existe depuis les internationales communistes. Le forum est en continuité dans la mesure où il tente de regrouper les initiatives qui souhaitent combattre la domination du monde par le capital et en rupture, parce qu'il cherche moins à trouver la ligne juste qui permet cette lutte qu'à mettre toutes les initiatives sur un pied d'égalité, dans une "horizontalité propositionnelle " ${ }^{3}$, afin que la communication entre celles-ci crée de nouvelles articulations porteuses de changement. Plutôt que de fixer un sujet abstrait unitaire dans l'appartenance à un parti relié directement à une condition sociale, le forum fixe l'unité du sujet de manière plus large, par son engagement dans des organisations (instances et mouvements) qui résistent et tentent de proposer des alternatives au capitalisme, à l'impérialisme, et à toute forme de domination d'un être humain sur un autre.

$\mathrm{Si}$ une telle rupture avec les mouvances internationalistes antérieures a été jugée nécessaire, c'est qu'une réponse unique au désastre du monde ne semblait plus possible. Du moins, cette réponse s'était vue reléguée aux abords de la poubelle de l'Histoire. Elle en était si près que les chantres du néolibéralisme clamaient qu'il n'y avait plus d'alternatives ${ }^{4}$ et que nous étions parvenus à la fin de l'Histoires. Elle leur permettait de dire que la société n'existe pas, seulement des individus isolés dont la responsabilité

$$
+4
$$

3. L'horizoncalité propositionnelle est le refus de hiérarchiser, au sein du forum, les propositions des différents groupes qui y participent. Tanc qu'elles sont en accord avec la charte de principe du forum, elles sont toutes traitées également, et le Conseil international se refuse généralement à adopter des positions politiques claires afin de maintenir cette horizontalité. C'est aux organisations participantes, et non au Forum, de faire la hiérarchisation des propositions en fonction de leurs préférences et analyses. Voir Chico WHITAKER, Changer le monde, [nouveau] mode d'emploi, Paris, Éditions de l'Atelier/Éditions Ouvrières, 2006 [2005], p. 50-54.

4. L'acronyme TINA est généralement reconnu pour résumer l'expression "There is no alternative" attribuée à Margaret Thatcher, premier ministre du Royaume-Uni de 1979 à 1990. Il signifie qu'il n'y a pas d'alternatives au virage économique qu'elle imposait à son pays, aujourd'hui connu sous le nom de néolibéralisme.

5. Référence au livre de Francis FuKUYAMA, La fin de l'Histoire et le dernier homme, Paris, Flammarion, 1992. 
principale est de s'occuper d'eux-mêmes ${ }^{6}$ : En plus d'être mal en point, la solution unique ne semblait plus suffisante pour bien des gens. Bien que le capitalisme demeure la forme d'exploitation centrale selon plusieurs organisations qui participent au Forum social mondial, la question de l'écologie, du racisme, des inégalités de genre et de sexe et de l'oppression des identités autochtones ne sont que des exemples d'une liste de problématiques qui s'allonge quotidiennement. Même si une bonne partie des groupes qui s'occupent de ces problématiques critiquent le néolibéralisme ambiant et le capitalisme dans son ensemble, ils parviennent difficilement à s'unir sous une seule et unique bannière, ne se reconnaissant pas dans la seule lutte des classes, et refusant le prolétariat comme sujet principal de l'Histoire. Comment, en effet, prôner le contrôle des usines par les ouvriers quand on veut en même temps diminuer la production pour sauver la planète? Comment demander aux peuples autochtones qui désirent préserver leur culture de se joindre à la lutte du prolétariat alors qu'ils ne souhaitent pas nécessairement s'intégrer à un système de production centralisée, tentant plutôt de préserver ce qui leur semble essentiel dans leurs pratiques culturelles ancestrales? Comment demander aux femmes d'attendre la révolution prolétarienne pour parvenir à une hypothétique égalité? Puisqu'une réponse simple à toutes ces questions était et demeure difficile à formuler, et est surtout peu susceptible de convaincre ceux et celles qu'elle aurait dû mobiliser, l'idée fut de simplement réunir toutes les réponses particulières à ces questions, et à bien d'autres, sous la bannière de ceux et celles qui croient qu'un autre monde est possible, que l'Histoire n'est pas terminée. Voilà l'origine du slogan " un autre monde est possible». Chico Whitaker, l'une des personnes à l'origine du Forum social mondial (FSM), disait en ce sens: "le forum ne cherche pas à créer une pensée hégémonique, mais à accepter la diversitế ."

\section{BRËVE HISTOIRE DES FORUMS SOCIAUX}

Afin de poursuivre cette brève présentation des principes ayant contribué à la formation des forums sociaux, il est nécessaire d'en étudier l'histoire d'un peu

$+4+$

6. On reconnaît ici la paraphrase d'une déclaration de Margaret Thatcher prononcée lors d'une entrevue accordée au magazine Woman's own: "TThere's no such thing as society. There are individual men and women and there are families. And no government can do anything except through people, and people must look after themselves first. It is our duty to look after ourselves and then, also, to look after our neighbours " (Douglas KEAY, "Aids, education and the year 2000 ", Woman's own, 31 octobre 1987, p. 8-10).

7. Les références à Chico Whitaker sont tirées d'une conférence qu'il a donnée le 27 août 2007 au centre St-Pierre à Montréal. La conférence avait pour titre: "Changer le monde. Nouveau mode d'emploi *. 
plus près. Bien que plusieurs interprétations existent au sujet de la naissance du Forum social mondial, les grandes lignes de son élaboration sont bien connues $^{8}$. La première édition s'est tenue à Porto Alegre en 2001, à l'appel de mouvements populaires brésiliens appuyés par quelques organisations étrangères dont plusieurs gravitaient autour du journal Le Monde diplomatique. L'une des origines du FSM se trouve dans la dynamique de contestation des négociations de traités commerciaux internationaux, alors appelée antimondialisation, qui s'exprimait par de grandes manifestations, dont celles contre la signature de l'Accord multilatéral sur les investissements (AMI) ${ }^{9}$, celle de Seattle (1999) contre l'Organisation mondiale du commerce et celle de Québec (2001) contre le Sommet des Amériques où se négociait la Zone de libre-échange des Amériques. À la même époque, des groupes organisaient des événements pour critiquer la tenue du Forum économique mondial (FEM), rendez-vous qui se tient annuellement à Davos et qui rassemble des gens d'affaires, des membres de gouvernements et des représentants d'institutions internationales. L'objectif du FEM est de lever les entraves à la croissance économique ${ }^{10}$, et il est perçu par ses opposants comme un endroit où les puissants travaillent à " perfectionner " le néolibéralisme" ${ }^{11}$. Le FSM fut d'abord construit comme un anti-FEM, c'est-à-dire une forme de vaste contestation de l'imposition d'un ordre économique qui délaisse de plus en plus les protections sociales pour un retour intensif aux règles du marché. Il était ainsi une critique de l'impuissance des États à résister aux pressions pour l'intégration dans un ordre international qui laisse peu d'espace à la régulation étatique, imposant plutôt une régulation externe sur laquelle les mouvements et instances de la société civile n'ont pas prise.

$$
+4
$$

8. Au sujer de l'histoire du Forum social mondial, voir notamment "Fórum Social Mundial", http://www.forumsocialmundial.org.br/index.php?cd_language=3 (28 septembre 2007); Bernard CASSEnS, Tout a commencé à Porto Alegre, Paris, Mille et une nuits, 2003.

9. De nombreuses manifestations ont eu lieu contre l'AMI, dans plusieurs pays qui devaient en être les signataires. Au Québec, en mai 1998, l'opération salAMI a regroupé quelques centaines de militants et militantes qui, par des actions de désobéissance civile, ont bloqué pendant quelques heures les rues autour de l'hôtel Sheraton où se déroulair la Conférence de Montréal (réunion du Forum économique international des Amériques). Cetre conférence accueillait alors le secrétaire général de l'Organisation de coopération et de développement économiques (OCDE), organisation qui regroupait les pays négociant l'AMI.

10. Sur le lien entre le FSM et le FEM, voir Dorval BRUNELLE, "Le Forum social mondial. Origine et participants", OBSERVATOIRE DES AMÉRIQUES, La Chronique des Amériques, no 3, janvier 2006. Aussi disponible en ligne : http://www.er.uqam.ca/nobel/ieim/article-oda.php3?id_article=2441 (28 septembre 2007).

11. Au centre de l'organisation de L'Autre Davos, la conférence alternative au FEM, on crouve l'organisation ATTAC-Suisse (http://www.otherdavos.net [17 novembre 2007]). 
Au niveau mondial, le forum social est coordonné par un Conseil international composé de plus de 130 organisations provenant de tous les continents. Sa composition est élargie par cooptation, et les barrières d'entrée sont très peu restrictives: il suffit que l'organisation qui veut y siéger adhère à la charte des principes. En son sein, les décisions se prennent par consensus des délégués, car le forum suit une démarche guidée par les principes d'écoute et de coopération, de respect de la diversité, plutôt que par ceux de compétition et de contrôle, qu'on estime plus caractéristiques du modèle capitaliste. Pour cette même raison, les rendez-vous du FSM ne se concluent pas par une déclaration finale officielle, et personne ne peut prétendre parler au nom de tous les participants et participantes. Le forum n'empêche pas les groupes d'émettre des déclarations en leur nom, et s'engage même à les diffuser, sans les hiérarchiser, en mentionnant le nom des organisations qui en assument les propos ${ }^{12}$. Celles-ci doivent évidemment respecter la charte des principes du FSM.

La première édition du Forum social mondial a regroupé 20000 participants et participantes ${ }^{13}$, alors que les organisateurs espéraient au plus réunir le tiers de cette foule. Lorsqu'il est devenu un rendez-vous annuel, le nombre de participants n'a cessé de croître, atteignant approximativement 155000 en $2005^{14}$. La rencontre annuelle a changé de continent, et s'est déroulée en Inde (2004) et au Kenya (2007). Sa forme a également évolué: il est devenu un forum polycentrique en $2006^{15}$. Avec une telle croissance, le FSM s'est rapidement détourné de son rôle d'antithèse du Forum économique mondial pour devenir un rassemblement et un processus pourvus d'une même méthodologie: créer un espace où, par l'horizontalité propositionnelle, on cherche à stimuler les articulations entre les organisations qui veulent bâtir un "autre" monde. À l'aide des forums, l'antimondialisation est devenue l'altermondialisation, et l'identité qu'elle développe ne

$$
+4
$$

12. CONSEIL INTERNATIONAL DU FORUM SOCIAL MONDIAL, "Charte des principes du Forum social mondial, article 7 ", http://www.forumsocialmundial.org.br/main.php?id_menu=4\&cd_language $=3$ (28 septembre 2007 ).

13. INSTITUTO BRASILEÑO DE ANÁLISIS SOCIALES Y ECONOMICAS, Foro social mundiah Rayo $X$ de la participación en el foro 2005 : elementos para debate, São Paulo, Secretaría internacional del FSM, 2006, p. 13.

14. Ibid.

15. Par polycentrique, les organisateurs définissaient la dynamique d'un forum se déroulant à peu près en même temps sur trois continents. L'édition de 2006 s'est ainsi déroulée à Bamako, à Caracas et à Karachj dans les premiers mois de l'année 2006 (FORUM SOCIAL MONDIAL, "Histoire du processus du FSM n, http://www.forumsocialmundial.org.br/main.php?id_menu=2\&cd_language=3 [28 septembre 2007]). 
correspond plus seulement à la critique de la mondialisation, mais aussi aux tentatives de créations d'alternatives.

La formule mondiale a fait naître des initiatives thématiques (Forum mondial de l'éducation) et régionales (continentales, souscontinentales, nationales, locales...) Suivant les mêmes principes, les forums régionaux cherchent à créer localement le même genre de pratiques que les éditions mondiales. La multiplication des initiatives locales fut tellement rapide que les membres du conseil international tentent aujourd'hui d'espacer les rendez-vous mondiaux afin de laisser plus de temps pour la réalisation d'initiatives à plus petite échelle ${ }^{16}$. Il fut ainsi décidé qu'en 2008 , le rendez-vous annuel n'aurait pas lieu, mais que les mouvements et instances qui participent à la dynamique du FSM étaient plutôt encouragés à organiser, dans leur pays respectif, une semaine d'action mondiale culminant par une journée globale de mobilisation et d'actions le 26 janvier.

\section{BREF HISTORIQUE DU FSQ}

Malgré les importantes délégations québécoises aux éditions mondiales ${ }^{17}$, la convocation d'un forum social au Québec ne fut pas une mince affaire. Plusieurs tentatives infructueuses ont précédé la réalisation d'un forum en 2007, dont celle, en 2003, qui proposait un forum social du Québec, du Canada et des premières nations ${ }^{18}$. Les efforts se sont multipliés (divisés), et des forums sociaux régionaux ont vu le jour dans la province, notamment pour la région de Québec/Chaudière-Appalaches (2002) et celle du Saguenay-Lac-Saint-Jean (2006). Toutefois, un événement d'envergure provinciale peinait à voir le jour. S'il est difficile d'identifier toutes les causes des échecs précédents, on peut néanmoins noter qu'une tentative en 2006 avait avorté principalement parce que les membres des différents comités ne parvenaient pas à mobiliser les organisations, instances et mouvements, ce qui " $[\ldots]$ a conduit à remettre en question le processus d'organisation,

$$
+4
$$

16. CONSEIL HÉMISPHÉRIQUE DES AMÉRIQUES DU FORUM SOCIAL MONDIAL, "Relatoria de la reunión de Liman, juillet 2006. Le compte rendu de la réunion du Conseil hémisphérique a été distribué par courriel aux gens qui étaient inscrits à la liste de diffusion du Conseil, dont l'auteur. Il n'est cependant pas archivé sur un sire Internet.

17. En 2005 , sur 150 pays représentés, le Canada figurait parmi les 10 pays ayant le plus de participants au forum, et parmi les 5 pays d'où provenait le plus grand nombre de participants au campement de la jeunesse (INSTITUTO BRASILENOO DE ANALISIS SOCLALES Y ECONOMICAS, op. cit., p. 16).

18. Voir Pierre BEAUDET, "Le Forum social mondial et l'altermondialisme ", Louis FaVreau, Gérald LAROSE et Abdou SALAM FALL (dir.), Altermondialisation, économie et coopétation internationale, Québec, Presses de l'Université du Québec, 2004, p. 303-315. 
jusqu'à présent majoritairement constitué de bénévoles se mobilisant sur une base citoyenne, de manière à donner une place plus importante aux organisation ${ }^{19} n$.

L'initiative de 2007 fut d'abord lancée par un collectif d'individus baptisé Initiative vers le $F S Q$ principalement composé d'étudiants ou de récents diplômés de l'Université du Québec à Montréal (UQAM) qui avaient participé aux éditions mondiales du forum. Ce collectif, bien que constitué de "bénévoles se mobilisant sur une base citoyenne ", est parvenu à regrouper suffisamment d'organisations québécoises (syndicats, organisations de solidarité internationale, associations étudiantes, groupes sociaux et communautaires...) pour tenir le premier forum social d'envergure provinciale.

Une tension entre les individus bénévoles et les organisations a néanmoins rapidement émergé. D'une part, les organisations ne pouvaient accepter que le processus de structuration du forum social accorde autant de poids décisionnel à des "bénévoles se mobilisant sur une base citoyenne" qu'à des représentants d'organisations coalisant un grand nombre de membres. Les organisations étaient les plus susceptibles de fournir le financement et les ressources nécessaires à la réalisation de l'initiative, tout en étant, contrairement aux bénévoles, redevables devant leurs membres. Par ailleurs, si les bénévoles réclamaient un pouvoir décisionnel, c'était précisément parce que les organisations n'avaient pas, jusque-là, assumé le rôle nécessaire à l'édification d'un processus menant à un forum social.

Le premier débat sur cette question a eu lieu lors de l'assemblée de fondation $^{20}$. Plusieurs propositions visant à avantager les organisations au détriment des bénévoles ont alors été rejetées. Les participants à l'assemblée sont parvenus à s'entendre sur un compromis quant à la distribution des droits de vote. Ainsi, un droit de vote a été accordé à chaque organisation impliquée, mais également à tous les membres du secrétariat ainsi qu'à un membre de chaque comité, qu'il soit d'organisation ou régional. Ainsi, les "bénévoles se mobilisant sur une base citoyenne" ont obtenu un certain pouvoir au sein du processus, mais uniquement par leur implication directe dans un comité d'organisation du Forum et par la reconnaissance de leurs pairs au sein de ce comité.

$$
++4
$$

19. L'Assemblée de fondation s'est tenue le 9 septembre 2006. Les procè-verbaux de toutes les assemblées sont disponibles en ligne: SECRETARIAT DU FSQ, "Forum social québécois n,

htcp:/www.er.uqam.ca/nobel/social/2007/publications/documentsorganisationnelsetprocesverbaux.html (28 seprembre 2007) [je souligne].

20. Ibid. 
Lassemblée préparatoire du 9 décembre 2006 s'est d'ailleurs terminée par une motion de félicitations aux bénévoles qui avaient porté le processus jusqu'à ce jour ${ }^{21}$. Au cours des différentes assemblées, la question de l'implication des organisations a resurgi à quelques reprises. Plusieurs membres du secrétariat, ces bénévoles à l'implication citoyenne, ont réussi à amasser les sommes nécessaires auprès des organisations pour que des salaires leur soient versés. Par contre, les organisations participantes, à une exception près, n'ont pas été capables de libérer des employés pour qu'ils participent directement à l'organisation de l'événement. Limplication citoyenne a donc été essentielle pour la réalisation du Forum social québécois.

\section{LE FSM ET LE FSQ, MEME COMBAT ?}

Pour le FSM, l'autre monde n'allait pas surgir de n'importe où et à l'initiative de n'importe qui. Pour trouver des pistes intéressantes et porteuses, il devait se bâtir sur l'expérience de ceux et celles qui s'organisent et luttent. Le forum devait ainsi permettre de créer une identité commune à tous ces mouvements, non pas sous une bannière fixe et déterminée, mais sous une bannière souple et floue, entraînant davantage l'inclusion que l'exclusion. Mais à vouloir trop inclure, ne risque-t-on pas d'oublier quelle est l'origine du propos: faire un autre monde?

Le pari de l'inclusion se faisait tout de même sur la base de deux constats qui rendaient nécessaire la création de l'espace du forum. D'une part, la montée du néolibéralisme mine la capacité des États à résister aux pressions mondiales qu'impose le marché. Par conséquent, la citoyenneté au sein des États peine à résister aux pressions mondiales puisque même les représentants élus et les assemblées législatives perdent leur pouvoir face aux exécutifs, d'abord, puis face aux organisations internationales et, finalement, aux pouvoirs privés, souvent du capital transnational ${ }^{22}$. D'autre part, contre cette perte de souveraineté, une foule de mouvements tente de résister à l'échelle locale, mais peine à le faire tant le pouvoir semble loin, perdu dans des sphères planétaires inaccessibles. C'est en ce sens que le FSM est d'abord apparu comme une antithèse du Forum économique mondial : il permettait

$$
+4
$$

21. lbid.

22. Au sujet du déficit démocratique, voir: Dorval BRUNELLE, op. cit. Le chapitre 6 traite spécifiquement de cette question. Pour prendre un exemple plus récent, il suffit de regarder comment se déroulent les négociations entre les chefs d'Étar du Mexique, du Canada et des États-Unis autour du Partenariat nordaméricain pour la sécurité et la prospérité (PSP), pour lesquelles le principal organe de consulcation est composé des chefs de 10 entreprises de chaque pays plutôt que de leurs instances législatives. 
de marquer une différence entre deux groupes, celui de Davos et celui de Porto Alegre.

Il devenait nécessaire de créer une citoyenneté mondiale à partir des organisations et des mouvements, non pas pour lutter contre un géant invisible, mais pour donner un levier, une force de justification internationale aux luttes locales. Malgré l'espace international de réalisation des premiers forums sociaux, l'objectif était, par la force de l'appui international, de renforcer les multiples luttes locales. Le FSM ne propose pas un repli étatique, mais bien la création d'une conscience planétaire pour façonner de nouvelles articulations entre les mouvements à l'échelle internationale, croyant notamment que celles-ci pourront soutenir plus efficacement les luttes et les alternatives qui se construisent au niveau local ${ }^{23}$.

Qui étaient les acteurs privilégiés de ces luttes? Il s'agit des organisations, des instances et des mouvements sociaux, définis de façon très large. On aurait pu appuyer les Etats directement, mais leur compromission - dont témoignent les pactes internationaux et la logique néolibérale mondialisée semblait déjà trop forte pour qu'ils puissent être la pierre angulaire de la résistance. Le FSM, en tant que réponse au FEM, dénonçait justement ce penchant des gouvernements à écouter davantage les grands patrons des multinationales que leurs propres citoyens. En fait, c'est justement contre la perte de pouvoir que se réunissaient les Forums. Quant aux partis politiques, leur compromission semblait égale. Les luttes pour gagner le pouvoir au sein des États ne sont pas à exclure complètement, mais peuvent également mener à des compromis et des culs-de-sac empêchant de nommer haut et fort la lutte qui est menée. Dans la société globalisée, les pressions sur les États sont telles que même les partis de gauche issus de guérillas se sentent parfois dans l'obligation de rassurer les investisseurs internationaux lorsqu'ils prennent le pouvoir ${ }^{24}$. Ils ne pouvaient donc non plus être l'élément fondamental de la résistance. Les simples citoyens, sans affiliation à une organisation, faisaient bien évidemment partie de la solution, mais pas en tant qu'individus. En effet, isolés, esseulés dans leur rapport à l'État, ils n'ont que peu de chances de résister. Au niveau mondial, les activités autogérées ${ }^{25}$ inscrites au forum

$$
4+4
$$

23. CONSEIL INTERNATIONAL DU FORUM SOCIAL MONDIAL, op. cit., article 14.

24. Ce fut notamment le cas de la guérilla maö̈ste népalaise, qui s'est assurée de ne pas faire fuir les investisseurs étrangers à la suite de ses récentes victoires.

25. La programmation d'un forum social est composée de divers éléments. Le comité organisateur se charge généralement d'organiser de grands panels sur des questions jugées primordiales avec quelques acteurs particuliètement pertinents. Toutefois, le corps de la programmation est composé d'activités 
par des individus, sans liens avec une ou des organisations, ne sont pas incluses au programme pour trois raisons. D'abord, on craint de donner une tribune à certains "fous" (comme le rappelait Chico Whitaker) et on suppose que le fait d'avoir un lien avec une organisation limite ce risque. Ensuite, on résout par là un problème logistique : le manque de salles disponibles pour réaliser toutes les activités que les quidams proposeraient. Mais surtout, on limite les conférences individuelles en soutenant qu'une idée partagée par un groupe, tels qu'une instance, une organisation ou un mouvement, a plus de chances d'avoir été débattue et travaillée, et d'être intéressante ou susceptible de produire des transformations sociales.

Il restait donc le recours aux organisations sociales (instances et mouvements), cette espèce de nébuleuse difficile à définir qui regroupe un peu de tout, des plus infortunés aux mieux organisés, des complètements exclus à ceux qui sont proches du pouvoir. La seule chose qui les unissait toutes était leur volonté de s'organiser afin de changer l'ordre des choses. C'est en ce sens que l'article 1 de la charte des principes énonce:

Le Forum social mondial est un espace de rencontre ouvert visant à approfondir la réflexion, le débat d'idées démocratique, la formulation de propositions, l'échange en toute liberté d'expériences, et l'articulation en vue d'actions efficaces, d'instances et de mouvements de la société civile qui s'opposent au néolibéralisme et à la domination du monde par le capital et toute forme d'impérialisme, et qui s'emploient à bâtir une societé planétaire axée sur l'être humain ${ }^{26}$.

Dans cet article, il n'est pas question de la participation individuelle ou citoyenne, mais bien d'instances et de mouvements. C'est à cette idée qu'a tenté de participer le FSQ. Il fut sans doute une réussite, avec ses 5000 participants, mais était habité d'une dérive qui le menace encore aujourd'hui : ce que nous appellerons la dynamique individuelle-citoyenne.

$$
+4
$$

autogérées (que les participants décident d'organiser eux-mêmes). Le comité organisateur se charge donc de recevoir les propositions, de trouver les endroits nécessaires a leur réalisation, et de les inscrire au programme. Il peut refuser les propositions qui ne respectent pas la charte, et suggérer la fusion d'activités ayant des thèmes semblables. Ces activités autogérées peuvent toucher à n'importe quel thème, mais le comité qui se charge de la programmation voit généralement à définir des axes qui permettent de regrouper les activités en fonction de leur thème. S'ajoutent à cela les activités culturelles, dont la programmation suit généralement le même principe que les activités autogérées, et quelques espaces définis pour traiter de problématiques particulières. Voir à ce sujet Chico WHITAKER, op. cit., p. 43-45. Il est également possible de consulter les programmes de plusieurs forums sociaux mondiaux: FORUM SOCIAL MONDIAL, "Memória do Fórum Social Mundial ",

http://www.forumsocialmundial.org.br/main.php?id_menu=14\&cd_language=1 (28 septembre 2007). 26. CONSEIL INTERNATIONAL DU FORUM SOCIAL MONDIAL, op. cit., article 1 [je souligne]. 
Lorsqu'on tente de la diviser, cette dynamique semble inoffensive. D'un côté, il y a l'individu, et il faut savoir que les forums sont toujours ouverts à quiconque veut $y$ assister. N'importe qui, en assistant un jour ou l'autre à une des activités, peut y voir des gens organisés qui mènent des projets pour changer le monde. On caresse l'espoir qu'il ou elle se joindra à un mouvement ou à une organisation pour faire avancer l'une ou l'autre des causes qui font partie du forum. Il est bien question de l'une ou l'autre des causes, puisque le pari du forum est justement de ne pas les hiérarchiser a priori. En les présentant toutes sur un pied d'égalité, on pense que les plus pertinentes coaliseront le plus de gens, et qu'alors, les nouvelles forces accumulées rendront cette lutte victorieuse. Mais surtout, en les mettant toutes sur un même pied, on espère que des luttes différentes vont trouver des points de convergence et s'articuler de façon à avoir plus de chances de gagner sur l'un et l'autre des fronts. Au FSQ, les inscriptions d'activités autogérées par des individus, sans l'appui d'une organisation, ont représenté approximativement $10 \%$ du total, ce qui n'est pas négligeable, sans être énorme non plus.

De l'autre côté, il y a la citoyenneté. L'idée prônée par le FSM est la création d'une citoyenneté planétaire, attendu que les luttes citoyennes dans chacun des pays doivent, pour avoir de meilleures chances de l'emporter, tenir compte des luttes qui se font ailleurs. Ainsi, la lutte pour de meilleures conditions de travail au Mexique devrait permettre de limiter l'érosion des emplois industriels au Nord, et la lutte contre l'implantation par une entreprise canadienne d'un oléoduc à travers les Andes aura d'autant plus de chances de gagner si cette compagnie sent qu'elle affronte non seulement la désapprobation de petites communautés autochtones perdues dans les montagnes, mais aussi celle de la population canadienne qui la perçoit comme une pollueuse et une exploiteuse sans vergogne ${ }^{27}$. Ici, la question de

$$
+4
$$

27. Mentionnons à cet égard la publication au Canada du livre d'Alain DENEAULT, Delphine ABADIE et William SACHER, Noir Canada. Pillage, corruption et criminalité en Afrique, Montréal, Edicions Écosociété, 2008. Les auteurs y critiquent, à l'aide de rapports publics, les actions de la compagnie aurifere Barrick Gold. Celle-ci a tenté d'en empêcher la publication par des menaces de poursuites et d'injonction. Une telle action défensive indique bien la sensibilité d'une compagnie à la mauvaise presse qui lui est faite dans son pays d'origine. Comme le suggère l'auteur du livre, la compagnie réagit probablement ainsi "[...] parce que les actifs des Canadiens se rrouvent à financer ces sociétés-là, par les fonds de retraite, les REER, les placements publics, les institurions financières, par l'entremise de la Bourse de Toronto." (SOCIETE RADIO-CANADA, "Barrick Gold. Controverse autour de Noir Canada ", http:/www.radiocanada.ca/nouvelles/societe/2008/04/15/002-barrick_noir-canada.shtml [29 avril 2008]). 
la citoyenneté implique surtout la dénonciation de la perte du rapport de forces des populations au sein de leur État respectif, et donc la nécessité de " transnationaliser" les luttes pour qu'elles aient un impact réel. En retour, la citoyenneté planétaire, par cette alliance internationale des luttes, donne plus de force aux citoyennetés locales.

Bien que ces deux aspects - individuel et citoyen - considérés séparément fassent bel et bien partie de l'idée générale qui constitue les forums sociaux, ils ont été joints dans la charte constitutive du FSQ, et représentent ainsi désormais un maillon faible de la dynamique que ce forum prétend créer. La première phrase du premier article de la charte stipule que le forum vise "l'implication citoyenne des individus ${ }^{28}$ ". Lorsque la lutte est décrite comme appelant d'abord l'implication citoyenne des individus, elle fait directement référence à l'individu dans sa relation avec l'État, à une masse informe et sans unité d'individus dont la seule existence en tant qu'ensemble est la somme des actions individuelles, sans plus. On croirait entendre Madame Thatcher. Contrairement à cela, les organisations cherchent à créer une identité collective, un sujet politique abstrait, exclu, pour lequel il est nécessaire de réformer ou de transformer complètement la citoyenneté. L'approche individuelle-citoyenne ne laisse comme identité que l'individu comme citoyen qui vote; que celui qui choisit ou non ses produits de consommation selon qu'ils polluent ou pas et selon le recyclage possible de son contenant; que celui qui se rassure en achetant du café équitable tout en oubliant que la cafetière utilisée provient d'un lieu de travail où les conditions sont encore plus désastreuses que celles des producteurs de café non équitable... Il ne reste que la conscience individuelle d'être contre le "système", et l'impression que la critique du téléjournal devant le téléviseur constitue un acte révolutionnaire.

L'approche individuelle-citoyenne semble faire fi de ce que sont les partis politiques : des organisations souvent très complexes dans lesquelles des débats et des votes président aux destinées de l'organisation (ou le devraient). Elle oublie que la pollution freinée par la consommation responsable est négligeable face au pouvoir de l'État de contraindre les entreprises à ne plus produire ou importer les contaminants ou les emballages inutiles. Elle ne dit pas non plus qu'une organisation est nécessaire pour la distribution du café équitable et qu'en plus, cette organisation impose bien souvent aux

$$
+\div
$$

28. SECRETARIAT DU FORUM SOCLAL QUÉbECOIS, "Chatte constitutive du Forum social québécois, Chapicre 1, art. 1.1.1", http://www.er.uqam.ca/nobel/social/2007/files/fsq-charte_constitutive.pdf (28 septembre 2007) [je souligne]. 
producteurs de telles denrées de s'organiser en coopérative pour avoir accès à ce réseau de distribution. La liste des exemples pourrait s'allonger, mais ceuxci semblent bien exprimer que pour transformer la citoyenneté défaillante du néolibéralisme, il faut s'organiser. Pour résister à cette idée d'une absence de société, il faut se redonner un pouvoir collectif face à l'application des seules règles du marché, dont la principale caractéristique est de faire gagner ceux qui sont déjà des gagnants, et de faire perdre les autres en leur laissant seulement l'espoir qu'un jour, eux aussi pourront s'enrichir s'ils réussissent à tirer profit du travail de leur voisin. En résumé, la logique individuellecitoyenne semble mettre de côté l'idée fondamentale que pour réformer la citoyenneté, il faut d'abord s'organiser.

Le FSQ aura malgré tout été un succès. Il a accueilli approximativement 5000 participants et participantes, alors que le Forum social d'un pays aussi peuplé que les États-Unis a réuni 10000 personnes. Il a permis de former de nouvelles coalitions et celles-ci ont organisé des manifestations dans le cadre du 26 janvier. Il n'en demeure pas moins une drôle d'impression. Dans les réunions d'organisation du forum, les assemblées préparatoires, des individus-citoyens réclamaient plus d'espace et la réalisation d'un second forum. Mais le but d'un FS n'est pas que des individus y voient un lieu d'organisation propre. Il est plutôt que ceux-ci côtoient des organisations et décident de s'y impliquer. C'est la caractéristique d'un forum d'être une espèce de foire aux alternatives pour que ceux et celles qui hésitent encore puissent se reconnaitre dans cette masse de gens qui veulent changer le monde. Et puisque le forum en lui-même n'est pas certain du mode d'emploi exact pour le changer, il propose l'éventail complet des solutions, afin que chacun y trouve son compte.

Surtout, une fois la première édition terminée, l'important n'est pas d'en réaliser une deuxième au plus vite pour les individus-citoyens qui n'ont pas saisi l'une ou l'autre des occasions au menu de la première. L'important est de s'assurer qu'à la prochaine édition le menu se sera élargi de nouveaux groupes et organisations de manière à multiplier d'autant les articulations.

Le poids de la responsabilité de cette dynamique ne peut incomber uniquement aux individus qui se sont impliqués dans l'organisation du FSQ. Les organisations sont également partiellement à blâmer. C'est ce qu'on comprend en lisant la déclaration de l'assemblée des mouvements sociaux. Les forums sociaux réservent généralement l'une des dernières plages horaires du programme à cette assemblée. Celle-ci n'est pas l'instance souveraine qui dirige le forum; elle permet plutôt la coordination des mouvements et 
organisations qui y participent, et la déclaration qui en résulte n'est pas la Déclaration du Forum, sinon celle des mouvements sociaux qui y prennent part. Cette frontière entre le Forum et l'assemblée des mouvements sociaux existe pour preserver l'aspect pluraliste du Forum. Ainsi, une organisation qui s'inscrirait en faux avec la déclaration de l'assemblée pourrait décider de continuer à participer au Forum tout en la dénonçant. Elle est toutefois la plus importante déclaration à émaner du processus, tant au niveau mondial que local.

La déclaration produite par l'assemblée des mouvements sociaux réunis en marge du FSQ débute par la phrase "[n]ous, membres des mouvements sociaux du Québec ${ }^{29}$ ". Alors que ce sont les organisations qui ont piloté le projet de déclaration, sans la participation des bénévoles-citoyens, cette première phrase met l'accent sur les individus qui participent aux mouvements plutôt que sur les mouvements eux-mêmes. Le ton généralement "personnaliste" de la déclaration abonde dans le même sens : il ést un aveu des quelques torts des militantes et militants des mouvements et une justification de ceux-ci formulés à la première personne du pluriel, ce même nous qui représente les membres des mouvements sociaux plutôt que les mouvements eux-mêmes. Ainsi, " toutes les avancées pour plus d'égalité, de justice et d'inclusion ont été le fruit de la ténacité de militantes et de mili$\operatorname{tants}{ }^{30}$ ", et non le fruit de leur coordination au sein d'organisations. L'appel se conclut logiquement par une invitation aux concitoyens et concitoyennes à se joindre à n'importe quel mouvement ou manifestation, il encourage les individus à faire partie de ce nous. Une rapide lecture des déclarations des assemblées du même genre tenues en marge des éditions mondiales montre qu'elles se présentent comme des déclarations des mouvements, et non des individus qui les composent, en conviant aux mobilisations à venir les organisations qui n'étaient pas au forum.

\section{DES PISTES POUR LES PROCHAINES EDITIONS}

Pour les prochaines éditions, il importe moins de laisser une place à chaque individu-citoyen qui voudrait exprimer ses sentiments face à la coordination du FSQ que d'insister sur la pertinence de charger les organisations et les instances de la coordination de l'événement. Il importe surtout de travailler

$$
+4
$$

29. "UniEs pour l'avenir du Québec et du monde", http://www.appelsolidaireduquebec.org/ spip.php?articlel (20 novembre 2007).

30. Jbid. 
à réunir les organisations qui n'ont pas participé à la première édition ${ }^{31}$. Par ce seul exercice, elles se rapprocheront et formuleront ainsi des pistes pour des articulations futures. C'est une solution sans garanties, mais dans le domaine du militantisme, contrairement à celui du marché, les garanties n'existent pas. Bien sûr, l'espace du forum doit demeurer ouvert pour que ceux qui veulent agir, sans savoir où, puissent magasiner les alternatives et y trouver le lieu qui leur convient le mieux. Néanmoins, plutôt que d'accepter que $10 \%$ des ateliers soient animés par des individus, il serait plus judicieux de les inviter à trouver une organisation qui accepterait d'endosser leur discours afin que les idées qu'ils veulent mettre de l'avant aient davantage de chances d'être débattues et partagées.

Cependant, quand il s'agit de l'assemblée des mouvements sociaux, la dynamique doit être différente. Elle n'est plus ce lieu où les individus magasinent, elle r'est plus le lieu de la foire où l'on invite tout le monde en criant : "Approchez ! approchez !", mais celui où les organisations doivent tenter de formuler une ligne d'action commune. Elle est le lieu où les luttes se rencontrent pour trouver de nouveaux points de convergence. Elle est l'embryon d'une hiérarchisation qui permet la coordination. La déclaration de l'assemblée des mouvements sociaux tenue en marge du FSQ n'invitait à aucune action particulière, elle axait plutôt ses efforts vers les individus n'appartenant pas aux mouvements. L'articulation des mouvements qui a résulté de l'assemblée est tout de même parvenue à coordonner la journée d'actions du 26 janvier 2008 appelée par le FSM, ce qui tend à démontrer que rien n'était perdu. Il est tout de même à espérer que la prochaine déclaration sera le fruit d'un effort supplémentaire pour coordonner et articuler les luttes. Sans devenir la coalition des coalitions dans un Québec où les mouvements sont déjà relativement réseautés, elle pourrait permettre des avancées supplémentaires, ou au moins, de freiner les reculs actuels.

En séparant les deux espaces de cette façon, on préserve la démarcation entre l'implication citoyenne des individus et la priorité accordée au sein des forums à la nécessité de l'organisation. Alors, cet autre Québec qui est en marche, selon le slogan du FSQ, pourrait passer au... trot.

$$
++4
$$

31. A cet égard, Diane Lamoureux notait l'absence de liens avec les milieux anglophones et le Canada anglais, ainsi que la faible présence des organisations autochtones (Diane LAMOUREUX, "Forum social québécois : quelles suites? », Relations, $n^{\circ} 721$, décembre 2007, p. 32-33). 